\title{
Quadrature Index Spatial Modulation with Two Constellations
}

\author{
Panmei Liu, a, Fuchun Huang ${ }^{2,3, b}$, Wen Zhao ${ }^{1, c}$ \\ 1. Department of Computer, College of Guangdong Peizheng, Guangzhou 510830, China; \\ 2. School of Electron and Information Engineering, Sun Yat-Sen University, Guangzhou 510275, \\ China; \\ 3. Department of Computer, South China Institute of Software Engineer GU, Guangzhou 510990, \\ China.
}

aliupanmei2006@126.com, bhfc_youyou@163.com, cpzcczw@foxmai.com.

\begin{abstract}
Quadrature index spatial modulation (QISM) with two constellations is presented to exploit the potential spatial diversity gain from spatial domain in multiple input multiple output (MIMO) channel system. In this paper, we consider a transmitted spatial vector formed by mapping two constellations symbol from BPSK and M-ary quadrature amplitude modulation (M-QAM/PSK) on the MIMO antenna array operating over Rayleigh fading channels and in the presence of additive white Gaussian noise. As a result, minimum Euclidean distance (MED) between the transmitted spatial vector in QISM scheme has a bigger spatial distance than that of quadrature spatial modulation (QSM). Then the bit error rate (BER) performance of QISM is analyzed. In addition, performance comparisons between spatial modulation (SM), QSM, QISM schemes for the different spectral efficiency are presented. The results demonstrate that our proposed scheme achieves the robust to the reliability in wireless communication network.
\end{abstract}

Keywords: Quadrature index spatial modulation, two constellations, minimum Euclidean distance.

\section{Introduction}

Quadrature spatial modulation (QSM) technology [1] has attracted significant attention from researchers due to its robustness to the reliability and the spectral efficiency. QSM retains all inherent advantage of the conventional spatial modulation (SM) and further exploit the spatial gain from the spatial domain of transmit antennas to carry more additional spatial antenna index bits. Thus providing better bit error rate (BER) than SM [2], V-BLAST [3] with the identical-throughput of QSM. In order to achieve the spatial diversity gain, in the GSM schemes proposed in [4]-[6], more than one antenna are activated at each time instant to transmit the same or different constellation symbols.

In addition, to further improve the spectral efficiency, Double Spatial Modulation (DSM) scheme [7] directly superimposes two independently SM transmission vectors by using constellation rotation.

Considering the previous schemes, a new scheme of quadrature index spatial modulation (QISM) with two constellations from BPSK and M-QAM/PSK is presented to further exploits the spatial diversity gain on increasing the minimum Euclidean distance (MED) between the spatial vectors.

The rest of this paper are organized as below. Firstly, Section 2 describes system model of the QISM scheme and ML detection, and making the performance analysis of QISM scheme in Section 3. Then the theoretical and simulation results are given in Sections 4. Finally, Section 5 concludes this paper.

\section{System Model}

\subsection{Transmitter of QISM Scheme}

We consider the QISM system with $N_{t}$ transmit and $N_{r} \quad$ receive antennas, as illustrated in Fig.1. Assuming that a total of $\mathrm{m}$ incoming data bits enters the transmitter of QISM. The $\mathrm{m}$ data bits are first divided into three blocks of data stream: data stream $B_{1}=\left\lfloor\log _{2} C_{N_{t}}^{2}\right\rfloor$ used for activating two transmit antenna, data stream $B_{2}=\log _{2} 2 M$ used for both BPSK and M-QAM/PSK symbols, and 
date stream $B_{3}=\log _{2} N_{t}$ used for activating one transmit antenna, where $\mathrm{M}$ denotes the modulation order of M-QAM/PSK .

For the block of data stream B1 bits, they are mapped on the index $\kappa_{i}$, two activated antennas of which

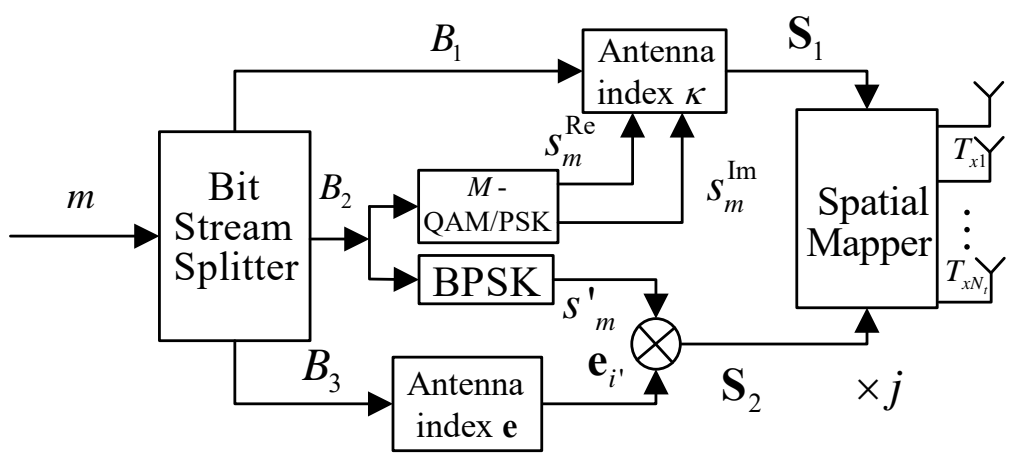

Fig 1. System model of QISM transmitter.

are used to transmit both BPSK and M-QAM/PSK symbols, from the index combinations of actvating two antenna $\kappa=\left\{\kappa_{1}, \kappa_{2}, \cdots, \kappa_{i}, \cdots, \kappa_{N_{\Omega}}\right\} \in \Omega, N_{\Omega}=2^{\left\lfloor\log _{2} C_{N_{t}}^{2}\right\rfloor}$, where $\Omega$ denotes the set of all possible antennas combinations $C_{N_{t}}^{2}$ for activating two antennas.

For the block of data stream $B_{2}$ bits are mapped onto one signal point $s_{m} \in\left\{s_{1}, s_{2}, \cdots, s_{m}, \cdots, s_{M}\right\}$ of M-ary QAM/PSK signal constellation and $S_{m}^{\prime}$ from BPSK symbols, respectively.

For the block of data stream $B_{3}=\log _{2}$ bits, they are mapped on the antenna index $e_{i^{\prime}}$, which drawn from the set with the cardinality $N_{t}$ as follows

$$
e=\left\{e_{1}, e_{2}, \cdots, e_{i^{\prime}}, \cdots, e_{N_{t}}\right\}
$$

where $e_{i^{\prime}} \in \mathbf{R}^{N_{t}}, i^{\prime}=1,2, \cdots, N_{t}$, is the $i^{\prime}$-th column of $\mathbf{I}_{N_{t}}$. In other words, only a single transmit antenna with index $i^{\prime}$ is activated to transmit a BPSK symbol at each time slot.

In the transmitter of QISM system, the $s_{m}^{\mathrm{Re}}$ and $s_{m}^{\mathrm{Im}}$ component of the resulting $s_{m}$ symbol are fed into the antenna index set $\kappa$, they are modulated on two different active antennas activated by B1 information bits and resulting in a spatial vector $\mathbf{S}_{1}$ which is considered as the real part of the transmitted spatial vector. Similarly, the resulting $S_{m}^{\prime}$ symbol is fed into the antenna index set $e$ module, then the $S_{m}^{\prime}$ is modulated on one activated antenna mapped by B3 information bits and resulting in the imaginary part $\mathbf{S}_{2}=S_{m}^{\prime} \cdot e_{i^{\prime}}$ of the transmitted spatial vector.

Finally, the transmitted complex spatial vector S can be obtained by adding S1 and S2 as follows.

$$
\mathbf{S}=\mathbf{S}_{1}+\mathrm{j} \cdot \mathbf{S}_{2}=\mathbf{S}_{1}+s_{m}^{\prime} \cdot e_{i^{\prime}}
$$

where $\mathrm{j}=\sqrt{-1}$ denotes the imaginary unit. 1.

To be more specific, the mapping rule of forming a transmitted spatial vector $\mathrm{S}$ is shown in Table

Thus, it can be seen that $\mathrm{B}=\mathrm{B} 1+\mathrm{B} 2+\mathrm{B} 3$ bits are transmitted simultaneously using QISM in $N_{t} \times N_{r}$ MIMO channel. Hence the transmitted maximum number of bits per QISM symbol can be calculated as

$$
R=\left\lfloor\log _{2} C_{N_{t}}^{2}\right\rfloor+\log _{2} 2 M+\log _{2} N_{t}
$$


Table 1. Examples of mapping $\mathrm{m}$ bits into a spatial vector $\mathrm{S}$

\begin{tabular}{|c|c|c|c|c|c|c|c|c|c|}
\hline \multicolumn{3}{|c|}{$m$ bits } & \multicolumn{7}{|c|}{ BPSK and 4QAM, $N_{t}=4$} \\
\hline$B_{1}$ & $B_{2}$ & $B_{3}$ & $\kappa_{i}$ & BPSK & 4QAM & $e_{i^{\prime}}$ & $\mathbf{S}_{1}$ & $\mathbf{S}_{2}$ & $\mathbf{S}$ \\
\hline 00 & 000 & 00 & $(1,3)$ & 1 & $(1,1)$ & $\begin{array}{c}{\left[\begin{array}{lll}1 & 0 & 0 \\
0 & 0\end{array}\right]^{\mathrm{T}}} \\
\end{array}$ & {$\left[\begin{array}{llll}1 & 0 & 1 & 0\end{array}\right]^{\mathrm{T}}$} & {$\left[\begin{array}{llll}1 & 0 & 0 & 0\end{array}\right]^{\mathrm{T}}$} & $\begin{array}{ccc}{\left[\begin{array}{ccc}1+\mathrm{j} & 0 & 1 \\
0\end{array}\right]^{\mathrm{T}}} & \\
\end{array}$ \\
\hline 01 & 001 & 01 & $(1,4)$ & 1 & $(1,-1)$ & $\begin{array}{c}{\left[\begin{array}{lll}0 & 1 & 0 \\
0 & 0\end{array}\right]^{\mathrm{T}}}\end{array}$ & {$\left[\begin{array}{llll}1 & 0 & 0 & -1\end{array}\right]^{\mathrm{T}}$} & {$\left[\begin{array}{lllll}0 & 1 & 0 & 0\end{array}\right]^{\mathrm{T}}$} & {$\left[\begin{array}{llll}1 & \mathrm{j} & 0 & -1\end{array}\right]^{\mathrm{T}}$} \\
\hline 10 & 010 & 10 & $(2,3)$ & 1 & 1) & $\begin{array}{c}{\left[\begin{array}{lll}0 & 0 & 1 \\
0 & 0\end{array}\right]^{\mathrm{T}}} \\
\end{array}$ & {$\left[\begin{array}{llll}0 & -1 & 1 & 0\end{array}\right]^{\mathrm{T}}$} & {$\left[\begin{array}{lllll}0 & 0 & 1 & 0\end{array}\right]^{\mathrm{T}}$} & {$\left[\begin{array}{llll}0 & -1 & 1+\mathrm{j} & 0\end{array}\right]^{\mathrm{T}}$} \\
\hline 11 & 011 & 11 & $(2,4)$ & 1 & $(-1,-1)$ & $\begin{array}{ccc}{\left[\begin{array}{lll}0 & 0 & 0 \\
1\end{array}\right.} & ]^{\mathrm{T}} \\
\end{array}$ & 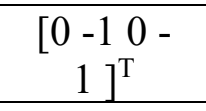 & {$\left[\begin{array}{lllll}0 & 0 & 0 & 1\end{array}\right]^{\mathrm{T}}$} & {$\left[\begin{array}{llll}0 & -1 & 0 & -1+\mathrm{j}\end{array}\right]^{\mathrm{T}}$} \\
\hline 00 & 100 & 00 & $(1,3)$ & -1 & $(1,1)$ & $\begin{array}{c}{\left[\begin{array}{lll}1 & 0 & 0 \\
0 & 0\end{array}\right]^{\mathrm{T}}} \\
\end{array}$ & {$\left[\begin{array}{llll}1 & 0 & 1 & 0\end{array}\right]^{\mathrm{T}}$} & {$\left[\begin{array}{llll}-1 & 0 & 0 & 0\end{array}\right]^{\mathrm{T}}$} & {$\left[\begin{array}{llll}1-\mathrm{j} & 0 & 1 & 0\end{array}\right]^{\mathrm{T}}$} \\
\hline 01 & 101 & 01 & $(1,4)$ & -1 & $(1,-1)$ & $\begin{array}{c}{\left[\begin{array}{lll}0 & 1 & 0 \\
0\end{array}\right]^{\mathrm{T}}} \\
\end{array}$ & {$\left[\begin{array}{llll}1 & 0 & 0 & -1\end{array}\right]^{\mathrm{T}}$} & {$\left[\begin{array}{llll}0 & -1 & 0 & 0\end{array}\right]^{\mathrm{T}}$} & {$\left[\begin{array}{llll}1 & -\mathrm{j} & 0 & -1\end{array}\right]^{\mathrm{T}}$} \\
\hline 10 & 110 & 10 & $(2,3)$ & -1 & $(-1,1)$ & $\begin{array}{c}{\left[\begin{array}{lll}0 & 0 & 1 \\
0 & l^{\mathrm{T}}\end{array}\right.} \\
\end{array}$ & {$\left[\begin{array}{llll}0 & -1 & 1 & 0\end{array}\right]^{\mathrm{T}}$} & {$\left[\begin{array}{llll}0 & 0 & -1 & 0\end{array}\right]^{\mathrm{T}}$} & {$\left[\begin{array}{llll}0 & -1 & 1-\mathrm{j} & 0\end{array}\right]^{\mathrm{T}}$} \\
\hline 11 & 111 & 11 & $(2,4)$ & -1 & $(-1,-1)$ & $\begin{array}{ccc}{\left[\begin{array}{lll}0 & 0 & 0 \\
1 & 1\end{array}\right]^{\mathrm{T}}} \\
\end{array}$ & 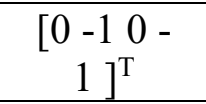 & $\begin{array}{c}{\left[\begin{array}{lll}0 & 0 & 0 \\
4 & 0\end{array}\right]^{\mathrm{T}}} \\
\end{array}$ & {$\left[\begin{array}{llll}0 & -1 & 0 & -1-\mathrm{j}\end{array}\right]^{\mathrm{T}}$} \\
\hline
\end{tabular}

\subsection{ML-Detection}

At the receiver, the received spatial signal at the receive antennas is expressed as:

$$
\mathbf{Y}=\rho \cdot \mathbf{H} \cdot \mathbf{S}+\mathbf{N}=\rho \cdot\left(\mathbf{h}_{\alpha}^{\kappa_{i}} \cdot S_{m}^{\mathrm{Re}}+\mathbf{h}_{, \beta}^{\kappa_{i}} S_{m}^{\mathrm{Im}}+j \cdot \mathbf{h}_{i^{\prime}}^{\mathbf{e}_{i}} \cdot S_{m}^{\prime}\right)+\mathbf{N}
$$

where $\mathbf{Y} \in C^{N_{r} \times 1}$ is a receiver signal vector, $\mathbf{H}=\left[\mathbf{h}_{1}, \cdots, \mathbf{h}_{i}, \cdots, \mathbf{h}_{N_{t}}\right] \in C^{N_{r} \times N_{t}}$ denotes the flatfading MIMO channel matrix, whose each entry being independent and identically distributed (i.i.d) complex-valued Gaussian random variables with $C N(0,1)$ and $\mathbf{N}$ with $\left(0, \sigma_{N}^{2} \mathbf{I}_{N_{r}}\right)$ is the additive noise vector. $\rho$ denotes the normalization $1 / \sqrt{E_{\mathbf{S}}}$ of the transmitted power. $\alpha, \beta \in\left\{1, \cdots, N_{t}\right\}$ are the row number of non-element in the index $\kappa_{i}$.

Assuming that the perfect channel state information (CSI) can be retrieved at the receiver, after the ML detection which performs the exhaustive search among the whole spatial symbol $\mathbf{S}$, the estimated indexes and symbols are obtained as

$$
\left[\hat{i}, \hat{i}^{\prime}, \hat{s}_{m}, \hat{s}^{\prime}{ }_{m}\right]=\arg \min \|Y-\rho \cdot f(\mathbf{S})\|^{2}
$$

where $\hat{i}, \hat{i}^{\prime}, \hat{s}_{m}, \hat{s}_{m}^{\prime}$ are the detected values, $f(\mathbf{S})=\mathbf{h}_{\alpha}^{\kappa_{i}} \cdot S_{m}^{\mathrm{Re}}+\mathbf{h}_{\beta^{K_{i}}}^{\kappa_{m}} S_{m}^{\mathrm{Im}}+j \cdot \mathbf{h}_{i^{\prime}}^{\mathbf{e}_{i}} \cdot S_{m}^{\prime}{ }_{m}$.

\section{Performance Analysis of QISM}

In this section, the BER performances are analyzed to demonstrate the outstanding performances of our proposed QISM scheme.

From [8], we can derive the union bound of the average bit error probability (ABEP) performances of QISM scheme from the expectation of the conditional pairwise error probability (CPEP).

With the assumption of perfect CSI, the CPEP can thereby be calculated by

$$
P(f(\mathbf{S}) \rightarrow f(\hat{\mathbf{S}}) \mid \mathbf{H})=Q\left(\sqrt{\frac{\rho}{2 \sigma^{2}} \cdot \sum_{r=1}^{N_{r}}\left|f_{r}(\mathbf{S})-f_{r}(\hat{\mathbf{S}})\right|^{2}}\right)
$$

where $\hat{\mathbf{S}}$ is the detected value, $Q(\cdot)$ denotes the Gaussian $\mathrm{Q}$ function.

The closed form expression for the expectation of the CPEP can be given as [8] 


$$
\bar{P}_{e}(f(\mathbf{S}) \rightarrow f(\hat{\mathbf{S}}))=\left(\frac{1-\mu}{2}\right) \sum_{r=0}^{N_{r}}\left(\begin{array}{c}
N_{r}-1 \\
r^{\prime}
\end{array}\right)\left(\frac{1+\mu}{2}\right)^{r^{\prime}}
$$

where $\mu=\sqrt{\frac{\gamma}{1+\gamma}}, \gamma$ is the mean of an exponential random variable.

Then ABEP of the QISM scheme can be derived as

$$
P_{b}=\frac{1}{m 2^{m}} \sum_{v=1}^{2^{m}} \sum_{w=1}^{2^{m}} \bar{P}_{e}\left(f_{v}(\mathbf{S}) \rightarrow f_{w}(\hat{\mathbf{S}})\right) \cdot e\left(f_{v}(\mathbf{S}) \rightarrow f_{w}(\hat{\mathbf{S}})\right)
$$

where $e\left(f_{v}(\mathbf{S}) \rightarrow f_{w}(\hat{\mathbf{S}})\right)$ is the total number of erroneous bits associated with the corresponding PEP event.

\section{Numerical Results and Discussions}

In this section, Monte Carlo simulations are performed to investigate the BER performances when the spectral efficiency and MIMO antenna number are different. Assumed that the transmitted spatial vectors over the flat Rayleigh distributed fading channel with $C N(0,1)$ and AWGN noise with $C N\left(0, \sigma^{2}\right)$.

Fig. 2(a) shows the theoretical ABEP performance at high SNR matches well with the simulated BER for the spectrum efficiency $\mathrm{R}=7 \mathrm{bits} / \mathrm{symbol}$ and transmit antenna number $\left[N_{t}, N_{r}\right]=[4,4]$, which verifies the effectiveness of our derivations. Moreover, it can be observed from the figure that QISM with 8-ary (4QAM, BPSK) has better BER performance as compared with SM and QSM schemes in scenarios of $\mathrm{R}=7 \mathrm{bits} / \mathrm{symbol}, \mathrm{R}=8 \mathrm{bits} / \mathrm{symbol}$ and the same transceiver antenna $\left[N_{t}, N_{r}\right]=[4,4]$. The BER performance of QISM with 16-ary (8QAM, BPSK) outperforms $2 \mathrm{~dB}$ SNR gains over the QSM scheme, and approximately 3.8 dB SNR gains over the SM schemes at BER value of 10-3 when $\mathrm{R}=7$ bits/symbol. Another scenario of $\mathrm{R}=8 \mathrm{bits} / \mathrm{symbol}$, more about $0.5 \mathrm{~dB}$ SNR gains than that of the QSM scheme and more $3 \mathrm{~dB}$ SNR gains than that of the SM scheme at BER value of 10-3 are achieved.

Furthermore, we compare the BER performances of QISM with that of other schemes. At both $\left[N_{t}, N_{r}\right]=[8,8]$ and $\mathrm{R}=10 \mathrm{bits} /$ symbol, as shown in Fig.2(b). Since exploiting spatial domain to transmitting more additional spatial index bits, it can be seen from the figure that QISM scheme achieves better performance than the other schemes such as SM, QSM. The QISM scheme can achieve $4 \mathrm{~dB}$ SNR gains than QSM, approximately $9 \mathrm{~dB}$ SNR gains than SM at BER value of 10-3. Therefore, the spatial domain in MIMO channel is worth of further investigating and exploiting. Similarly, on condition that $\left[N_{t}, N_{r}\right]=[8,8]$ and $\mathrm{R}=11$ bits/symbol, QISM scheme has better performance than SM, QSM.
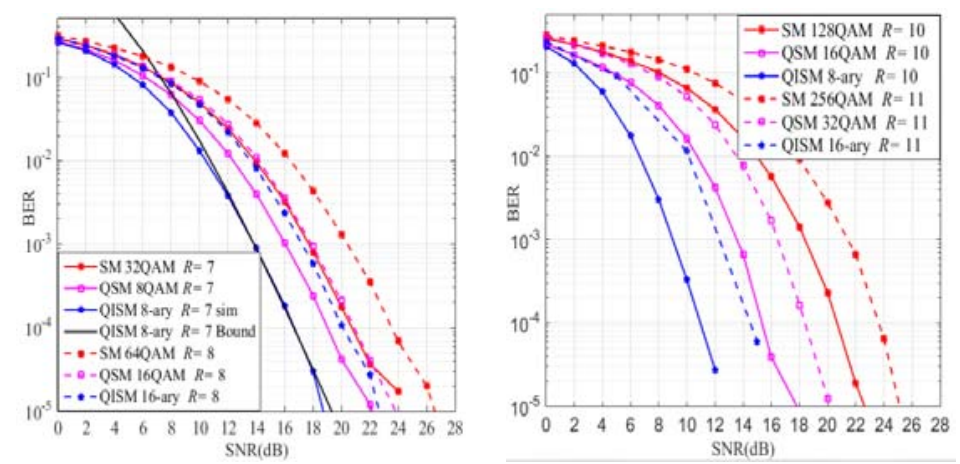

(a) BER comparison of SM, QSM, QISM with different modulation rate (b) BER performance comparison among QISM, SM, QSM

Fig 2. BER performance of QISM with various schemes 


\section{Conclusion}

In this paper, we propose a new scheme of QISM structure with two constellations to further exploit the spatial domain for enhancing the reliability of the MIMO-based communication system. Owing to improving the squared MED between the spatial vectors, our present scheme achieve better BER performance than other schemes. Furthermore, the BER performance is analyzed. Finally, the numerical analysis results demonstrate that our proposed scheme improves significantly the performances of the MIMO-based wireless systems.

\section{References}

[1]. R. Mesleh, S. S. Ikki, and H. M. Aggoune, "Quadrature spatial modulation," IEEE Trans. Veh. Technol, vol. 64, no. 6, pp. 2738-2742, June 2015.

[2]. R. Mesleh, H. Haas, S. Sinanovic, et. al., "Spatial modulation," IEEE Trans. Veh. Technol, vol. 57, no. 4, pp. 2228-2241, July 2008.

[3]. P. Wolniansky, G. Foschini, G. Golden, et. al., "V-BLAST: an architecture for realizing very high data rates over the rich-scattering wireless channel," in Proc. International Symposium on Signals, Systems, and Electronics (ISSSE’98), Pisa, Italy, Sept. 1998, pp. 295-300.

[4]. A. Younis, N. Serafimovski, R. Mesleh, et. al., "Generalised spatial modulation," in Proc. 2010 Forty Fourth Asilomar Conference on Signals, System and Computers, Nov. 2010, pp. 14981502.

[5]. J. Wang, S. Jia, and J. Song, “Generalised spatial modulation system with multiple active transmit antennas and low complexity detection scheme," IEEE Trans. Wireless Commun, vol. 11, no. 4, pp. 1605-1615, Mar. 2012.

[6]. T. Datta, H. S. Eshwaraiah, and A. Chockalingam, "Generalized Space-and-Frequency Index Modulation,” IEEE Trans. Veh. Technol, vol. 65, no. 7, pp. 4911-4924, July 2016.

[7]. Z. Yigit and E. Basar, "Double spatial modulation: A high-rate index modulation scheme for MIMO systems," in 2016 International Symposium on Wireless Communication Systems (ISWCS), Sept. 2016, pp. 347-351.

[8]. M. K. Simon and M. Alouini, Digital communication over fading channels. Wiley, USA: Wiley Series in Telecommunications and Signal Processing, 2nd edition, 2005. 